\title{
Monitoring Neuromuscular Blockade
}

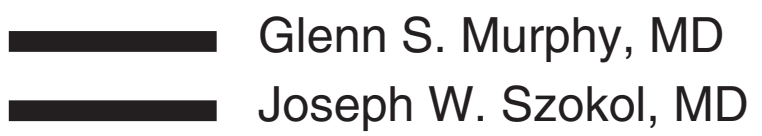

Neuromuscular-blocking agents (NMBA) have been used in clinical anesthesia for nearly 60 years. In the perioperative period, a variety of factors can influence a patient's response to these drugs. To precisely titrate NMBAs to clinical effects in the anesthetized patient, the application of neuromuscular monitoring is essential. The use of a peripheral nerve stimulator allows the clinician to assess the intensity of the neuromuscular block. Profound muscle relaxation (to permit tracheal intubation and ensure immobility), moderate muscle relaxation (to facilitate surgical procedures), and recovery of neuromuscular function can be evaluated by monitoring evoked responses to nerve stimulation. This chapter reviews the physiology of neuromuscular transmission, the fundamental principles of neurostimulation, the patterns of nerve stimulation used in the operating room, and the assessment of evoked responses to peripheral nerve stimulation. In addition, the role of neuromuscular monitoring in detecting, and possibly avoiding, residual paralysis in postoperative patients is examined.

\section{- Physiology Of Neuromuscular Transmission}

The neuromuscular junction consists of the motor nerve terminus and the end plate of the muscle fiber (Fig. 1). These 2 structures are separated by a narrow gap called the synaptic cleft. Acetylcholine (ACh) released from the motor nerve terminus is able to rapidly transverse the 20- to 50-nm synaptic cleft and bind with specialized receptors on the postsynaptic end plate. 


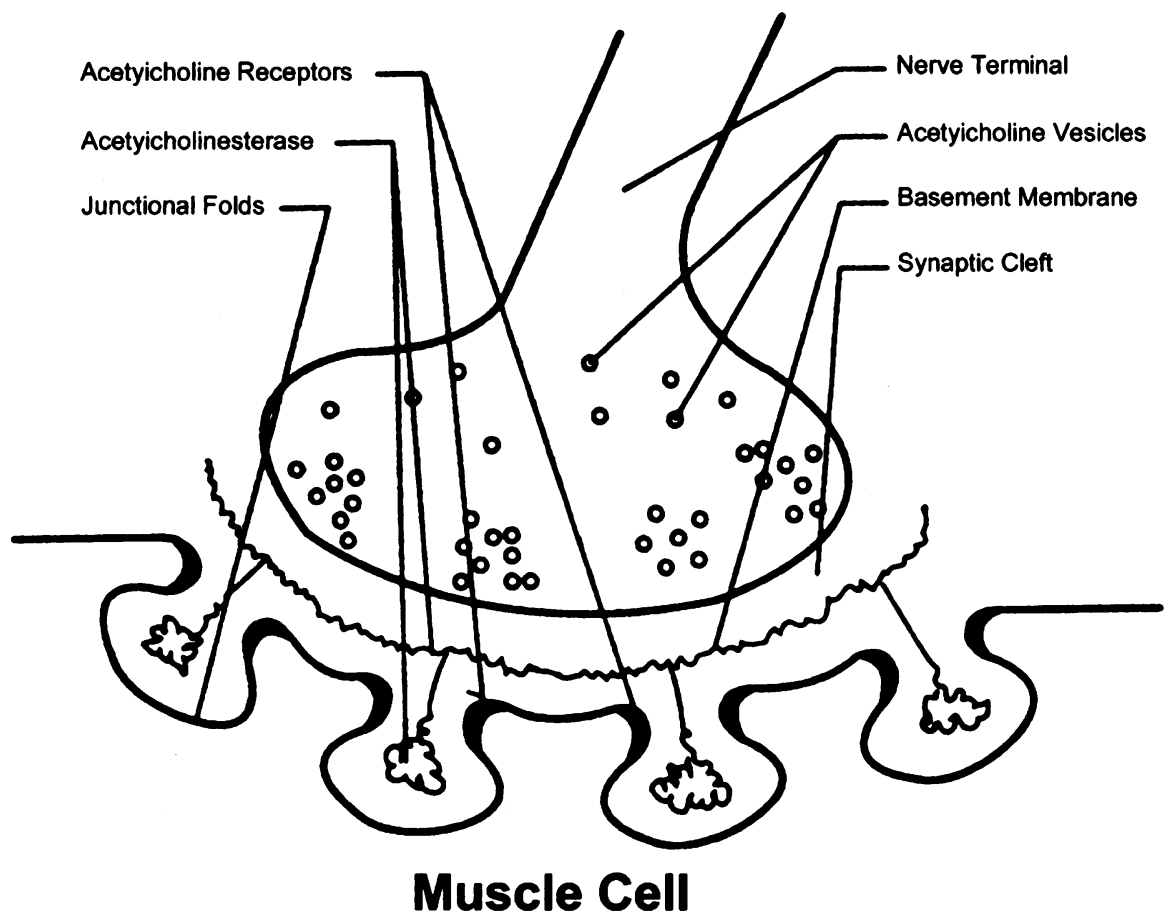

Figure 1. Diagram of the neuromuscular junction. Reproduced with permission from Bevan DR, Donati F. Muscle relaxants. In: Barash PG, Cullen BF, Stoelting RK, eds. Clinical Anesthesia, ed 4. Philadelphia: Lippincott Williams and Wilkins; 2001:419-447.

\section{Motor Nerve Terminus}

Acetylcholine is synthesized in the axon terminal of motor nerves from choline and acetate. It is stored in small vesicles, or quanta, with each vesicle containing approximately 5000 to 10,000 molecules of Ach. Acetylcholine in the nerve terminal is available in 2 fractions: a small, immediately available pool stored in vesicles, and a larger reserve pool found both free in the cytoplasm and in vesicles. ${ }^{1}$ When an action potential reaches the nerve terminus, voltage-responsive ion channels open and calcium influx occurs. This results in fusion of 200 to 400 vesicles from the immediate pool with the axonal membrane and release of ACh into the synaptic cleft. ${ }^{2}$ High-frequency stimulation of motor nerves can produce depletion of the immediately available stores of ACh. The rate of ACh release decreases until an equilibrium is reached between mobilization of ACh from the reserve pool and release of ACh from the immediate stores. Normal muscular contraction occurs with high-frequency nerve stimulation, because far more $\mathrm{ACh}$ is released than is required to produce a full muscular response (a "wide margin of safety"). In the presence of a partial nondepolarizing neuromuscular block, the number of free nicotinic ACh 
receptors on the postsynaptic membrane is reduced. This reduces the margin of safety of neuromuscular transmission, and a reduced force of muscular contraction can be measured. Fade is the term used to describe this progressive decrease in muscle response to rapid, repetitive nerve stimulation. Fade can be observed when patients are monitored with trainof-4, tetanic, or double-burst stimulation modes. Muscle relaxants can also produce fade by blocking presynaptic cholinergic receptors, which play a role in the mobilization of ACh in motor neurons. ${ }^{3}$ High-frequency stimulation of motor nerves also facilitates mobilization of ACh from the reserve pools to the immediate stores and increases the synthesis of ACh from choline and acetate. ${ }^{4}$ This enhanced synthesis and mobilization of ACh explains the phenomenon of posttetanic potentiation. If a single twitch stimulus is provided within 2 minutes of a 5-second tetanic stimulus, an exaggerated evoked muscular contraction will occur.

\section{Motor End Plate Region}

Millions of nicotinic acetylcholine receptors (nAChR) are localized at the end plate region of the muscle fiber. Each receptor is composed of 5 glycoprotein subunits ( 2 alpha, 1 beta, 1 delta, and 1 epsilon) arranged around a central channel (Fig. 2). When 2 ACh molecules bind simultaneously to both of the alpha subunits, an ion channel in the center of the receptor opens, and sodium ions move inside the cell. A muscle action potential occurs when $10 \%$ to $20 \%$ of the receptors are open on a motor end plate. The depolarization spreads throughout the muscle fiber and the fiber then contracts. The force of contraction of a muscle is directly related to the number of muscle fibers that are activated. Nondepolarizing muscle relaxants produce neuromuscular blockade by binding to the al-
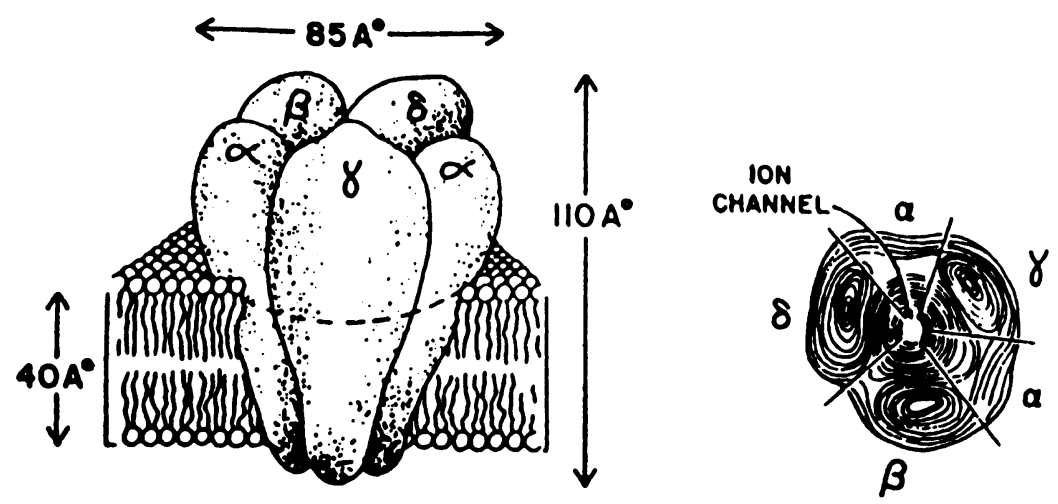

Figure 2. Diagram of the nicotinic acetylcholine receptor. Reproduced with permission from Taylor P. Are neuromuscular blocking agents more efficacious in pairs? Anesthesiology. 1985;63:1-6. 
pha subunits of the postsynaptic nAChR. These agents have no agonist activity at the nAChR and result in a competitive block by preventing ACh from binding to the receptor and activating a muscle action potential. Nondepolarizing NMBAs can also produce their effects by physically occluding or blocking the ion channel of the receptor ("channel block"). Depolarizing agents (succinylcholine and decamethonium) bind to the $\mathrm{nAChR}$ and produce an agonist response. Because succinylcholine is not metabolized by acetylcholinesterase at the neuromuscular junction, it can bind repeatedly to the nAChR, resulting in persistent end plate depolarization and flaccid paralysis.

\section{- Fundamental Principles Of Neurostimulation}

For clinicians to properly use peripheral nerve stimulators, an understanding of the following concepts is required.

\section{Current}

Muscle contraction in response to nerve stimulation is dependent on the current delivered by the stimulator. The amount of current required to elicit a detectable muscle response is the threshold current. When using surface electrodes, this value is approximately $15 \mathrm{~mA} .^{5}$ The current needed to induce depolarization in all of the fibers in a nerve bundle is the maximal current. For clinical monitoring, a supramaximal current is usually applied, which is $10 \%$ to $20 \%$ greater than the maximal current and 2 to 3 times higher than the threshold current. ${ }^{6}$ A 50 to $60 \mathrm{~mA}$ current across surface electrodes is required to produce a supramaximal response, which can be painful in the awake patient.

\section{Resistance}

The force opposing the flow of energy between the electrodes of the stimulator and the peripheral nerve is the resistance. Because current is equal to voltage divided by the resistance, any increase in tissue resistance will necessitate a proportional increase in voltage to maintain a constant stimulating current. Most peripheral nerve stimulators will deliver a constant current over a range of changing resistances by varying the internal voltage. Resistance between the surface electrodes and the skin can be reduced by removing excess hair, decornifying and degreasing the skin, and cleaning the area beneath the electrodes with alcohol. If tissue resistance prevents the stimulating current from reaching the nerve (ie, in the morbidly obese patient), needle electrodes can be used instead of gelcovered conducting surface electrodes. Currents $\leq 10 \mathrm{~mA}$ will usually produce supramaximal stimulation when subcutaneous needle electrodes are used. $^{7}$ 


\section{Pulse Duration/Wave Form}

The duration of each impulse delivered by the stimulator is ideally between 0.2 to $0.3 \mathrm{~ms}$. If the impulse duration is greater than $0.5 \mathrm{~ms}$, repetitive nerve firing or direct muscle stimulation can occur. The waveform should be monophasic and square wave so that a constant current is maintained over the entire impulse. This avoids repetitive nerve stimulation, which will enhance the twitch response.

\section{Frequency of Stimulation}

The rate (in cycles/second or Hertz $[\mathrm{Hz}]$ ) at which impulses are delivered by the peripheral nerve stimulator is the stimulus frequency. Single-twitch stimulation usually involves a stimulus frequency of either one impulse every 10 seconds $(0.1 \mathrm{~Hz})$ or one impulse every second (1 $\mathrm{Hz}$ ). Train-of-4 monitoring consists of the application of 4 supramaximal stimuli to the nerve in 2 seconds $(2 \mathrm{~Hz})$. Tetanic stimulation requires the delivery of a very high frequency of electrical stimuli $(30-100 \mathrm{~Hz})$. In clinical practice, the most commonly used pattern of tetanic stimulation is a $50-\mathrm{Hz}$ impulse given for 5 seconds.

\section{Electrode Placement}

Optimal nerve stimulation occurs when the negative electrode is placed directly over the nerve. The positive electrode is usually placed proximally to the negative electrode to avoid depolarizing a different nerve. For ulnar nerve stimulation, the negative electrode should be placed proximal to the skin crease at the wrist on the radial side of the flexor carpi ulnaris tendon. The positive electrode can be positioned $3 \mathrm{~cm}$ proximally to the negative electrode or at the ulnar groove at the elbow.

\section{- Patterns Of Nerve Stimulation}

Four patterns of nerve stimulation are commonly used by clinicians in the perioperative setting: single twitch, train-of- 4 , tetanic and posttetanic count, and double burst stimulation.

\section{Single Twitch}

This is a simple form of monitoring in which a single supramaximal stimulus is applied for 0.1 to $0.3 \mathrm{~ms}$. Twitch height will remain normal until $75 \%$ of the nAChRs are blocked and will completely disappear when $90 \%$ to $95 \%$ of the nAChRs are occupied. ${ }^{8}$ There are limitations associated with single-twitch monitoring. A controlled twitch height must be 
measured before muscle relaxants are administered, and specialized recording devices are needed to compare the subsequent twitch heights to the control height. In addition, the response to stimulation is frequencydependent. At rates of $0.1 \mathrm{~Hz}$ (once every 10 seconds), there is no impact on neuromuscular transmission. When rates are increased to $1.0 \mathrm{~Hz}$ (once every second), fade will be observed, and a faster onset of neuromuscular block can develop in the stimulated muscles. ${ }^{9}$

\section{Train-of-Four}

Since its introduction into clinical practice in the early 1970s, trainof-4 (TOF) monitoring has become the most widely used mode of neurostimulation. Four supramaximal stimuli are applied to a peripheral nerve over a 2-second interval. The observed response can be quantified as a TOF count or a TOF ratio. The TOF count is a simple assessment of the number of twitches (0-4) after TOF stimulation. The TOF ratio is a measure of the amplitude of the fourth twitch $\left(\mathrm{T}_{4}\right)$ divided by the amplitude of the first twitch $\left(\mathrm{T}_{1}\right)$. The determination of accurate TOF ratios requires specialized recording equipment such as mechanomyography, electromyography, or acceleromyography. The TOF count is commonly used in the operating room and intensive-care unit because it is simple to perform and can be quantified without additional recording devices. In the absence of muscle relaxants, the TOF ratio is 1.0 in normal subjects. During a partial nondepolarizing block, a progressive decrease in the amplitude of each twitch (fade) will be observed. When $70 \%$ to $75 \%$ of the nAChRs are occupied, the height of $\mathrm{T}_{4}$ begins to decrease. The amplitude of $\mathrm{T}_{1}$ is reduced by $75 \%$ when $80 \%$ of the receptors are blocked. When $85 \%, 85 \%$ to $90 \%$, and $90 \%$ to $98 \%$ of the nAChRs are occupied, the T3, $\mathrm{T} 2$, and $\mathrm{T} 1$ responses are abolished, respectively. ${ }^{10}$ Train-of-4 monitoring has many advantages over other patterns of nerve stimulation. The TOF count or TOF ratio can be used to evaluate the intensity of the neuromuscular block, particularly when evoked responses are measured with recording equipment. Unlike single-twitch monitoring, TOF monitoring does not require the recording of a control response; $\mathrm{T}_{4}$ is compared with $\mathrm{T}_{1}$, not with a control measurement. Studies have demonstrated that the TOF ratio is more sensitive in detecting subtle degrees of neuromuscular blockade than single-twitch stimulation. ${ }^{11}$ TOF monitoring can be performed relatively frequently (once every 10-12 seconds) without influencing twitch height. This is in contrast to tetanic stimulation, which can only be performed once every 2 minutes. An exaggerated response to singletwitch and TOF monitoring will occur if either is administered within 1 to 2 minutes of a tetanic stimulation as a result of posttetanic potentiation. In addition, TOF monitoring is much less painful than tetanic stimulation, especially when submaximal currents are used. Therefore, the TOF response can be used to detect residual block in the awake patient. 


\section{Tetanic Stimulation and Posttetanic Count}

When motor nerves are stimulated at rates of greater than $30 \mathrm{~Hz}$, the twitch responses fuse, and a single sustained contraction of the muscle occurs. Most peripheral nerve stimulators are designed to deliver a $50-\mathrm{Hz}$ tetanic stimulus. The muscle tension that develops when a peripheral nerve is stimulated at this frequency is equivalent to the tension that is observed during maximal voluntary effort. ${ }^{12}$ Tetanic stimulation at supraphysiological rates of 100 to $200 \mathrm{~Hz}$ can produce fade in anesthetized patients in the absence of NMBAs. ${ }^{13}$ Therefore, frequencies greater than $50 \mathrm{~Hz}$ should not be used in clinical practice. If more than $70 \%$ to $75 \%$ of the nAChRs are blocked by a nondepolarizing muscle relaxant, fade will occur during titanic stimulation. The degree of fade can be used like the TOF ratio to estimate the intensity of the neuromuscular block. The primary disadvantage of tetanic monitoring is that it is extremely painful and should only rarely be used in awake patients. Another disadvantage is that high-frequency stimulation can antagonize neuromuscular block in the muscle that is being monitored. ${ }^{14}$ This can complicate interpretation of subsequent responses. When a single-twitch stimulus is administered within 2 minutes of a tetanic stimulus, an enhanced response is observed as a result of posttetanic potentiation. A posttetanic count (PTC) typically involves the application of a $50-\mathrm{Hz}$ tetanic stimulation for 5 seconds, followed 3 seconds later by a single supramaximal stimulus delivered once every second. Posttetanic count can be used to evaluate intense neuromuscular block when no response to single-twitch or TOF stimulation can be measured. This mode of monitoring is used when profound muscle relaxation is required, and complete immobility of the patient is essential for optimal care (ie, during ophthalmic surgery on an open globe, or to prevent coughing during tracheal suctioning in patients with elevated intracranial pressure). Posttetanic count is also used to estimate the time to recover from intense neuromuscular block. When a PTC of 1 is detected in patients who have received pancuronium, approximately 30 to 40 minutes will elapse before the first response $\left(T_{1}\right)$ to TOF stimulation is observed. ${ }^{15}$ During vecuronium or atracurium neuromuscular blockade, a PTC of 1 will predict the return of $\mathrm{T}_{1}$ within 7 to 8 minutes. ${ }^{16,17}$ The PTC correlates inversely with the time required for return of neuromuscular function (a higher number of evoked responses predicts a more rapid return of $\mathrm{T}_{1}$ ). The PTC is useful in determining when a profound neuromuscular block can be reversed, because this should not be attempted until at least the first response to TOF stimulation has returned.

\section{Double-Burst Stimulation}

Double-burst stimulation (DBS) was developed to allow the clinician to detect subtle degrees of neuromuscular blockade without the use of recording devices. Two short bursts of tetanic stimuli, delivered at a fre- 
quency of $50 \mathrm{~Hz}$, are separated by a 750-ms interval. Each burst consists of a series of 3 and 2 impulses $\left(\mathrm{DBS}_{3,2}\right)$ or 3 and 3 impulses $\left(\mathrm{DBS}_{3,3}\right)$. A good correlation between the ratios of the second burst to the first $\left(\mathrm{D}_{2} / \mathrm{D}_{1}\right)$ and the $\mathrm{T}_{4} / \mathrm{T}_{1}$ ratio has been demonstrated. ${ }^{18}$ Clinical studies have also indicated that fade is more easily detected with DBS than with TOF stimulation. When a manual evaluation of TOF responses is used, fade can only be reliably detected when the TOF ratio is $<0.4 .^{19,20}$ In contrast, manual assessment of evoked responses to $\mathrm{DBS}_{3,3}$ allows for the detection of fade up to TOF ratios of $0.6 .^{19}$

\section{- Assessment Of Responses To Nerve Stimulation}

\section{Visual or Tactile Assessment}

A visual or manual evaluation of muscle contraction in response to nerve stimulation is the most common method used to monitor evoked responses. Unfortunately, significant residual muscle weakness might not be detected when this method is used. As stated previously, most clinicians are unable to assess the presence of fade by visual or tactile means when the TOF ratio is as low as 0.4 . If no TOF fade is manually detected by experienced clinicians, significant residual neuromuscular block could still be present in up to $50 \%$ of patients. ${ }^{19}$ The use of a DBS pattern of stimulation could improve the detection of residual paresis in the perioperative period.

\section{Mechanomyography}

Mechanomyography objectively measures isometric contraction of the adductor pollicis muscle in response to ulnar nerve stimulation. The force of contraction is converted into an electrical signal and recorded. To accurately record responses, a 200-to 300-g preload must be attached to the thumb, the hand must be immobilized, and the force transducer must be aligned with the direction of the movement of the thumb. Although mechanomyography is considered the "gold standard" in assessing evoked responses, it is rarely used in clinical practice.

\section{Electromyography}

Electromyography (EMG) measures the electrical activity, the compound muscle action potential, of the stimulated muscle. The 2 active electrodes are placed over the body and tendinous insertion of the monitored muscle, and a third neutral electrode is positioned at a remote site. After nerve stimulation, EMG responses are typically measured from the thenar eminence, the hypothenar eminence, or the first dorsal interosse- 
ous muscles of the hand. Electromyography units calculate the amplitude of the signal, which represents the sum of the individual compound muscle action potentials, or the area under the EMG curve. ${ }^{21}$ A relatively good correlation between measured evoked responses obtained with EMG and mechanomyography has been demonstrated. ${ }^{22}$ Small differences in response occur, however, because EMG is measuring electrical activity and mechanomyography is recording contraction of the muscle. The quality of the EMG signal can be adversely affected by a number factors, including electrical interference, improper electrode placement, direct muscle stimulation, and hypothermia.

\section{Acceleromyography}

Newton's second law states that force $=$ mass $\times$ acceleration. If mass is constant, the force of a muscle contraction can be calculated if acceleration is measured. Accelerometers use a small piezoelectric transducer, which is attached to the stimulated muscle. The movement of the muscle generates a voltage in the piezoelectric crystal, which is proportional to the acceleration of that muscle. The signal is rapidly analyzed and the results displayed. Acceleromyography was introduced primarily for use during clinical anesthesia. The devices are small, portable, and easy to use. Because isotonic contraction is measured, no preload needs to be placed on the stimulated muscle. This allows the clinician to monitor neuromuscular blockade at sites in which mechanomyography cannot be used such as the orbicularis oculi. Clinical studies have demonstrated a good correlation between TOF ratios obtained with acceleromography and those measured by mechanomyography. ${ }^{23,24}$ Because acceleromyography is so easy to use, it offers promise as a reliable monitor to detect residual neuromuscular block in the operating room or postanesthesia care unit.

\section{Acoustic Myography}

A new method to evaluate evoked responses has been recently described. Acoustic myography is based on the principle that contraction of skeletal muscles generates low-frequency sounds that can be detected with a low-frequency microphone. Preliminary studies have suggested that the clinical performance of acoustic myography was comparable with that of mechanomyography, electromyography, and acceleromyography. ${ }^{25,26}$

\section{Neuromuscular Monitoring In The Operating Room}

\section{Sites of Nerve Stimulation}

In the perioperative setting, the ulnar nerve at the wrist is the most popular site for neurostimulation, and the response at the adductor pollicis is observed or recorded. During many surgical procedures, the pa- 
tient's arm is positioned in a manner in which it is not accessible to the clinician for monitoring purposes. In this situation, the facial nerve is often used as a monitoring/stimulation site, and the contraction of the muscles around the eye is then evaluated. When interpreting the motor response to peripheral nerve stimulation, it is important to understand that different muscles have different onset times, offset times, and sensitivities to muscle relaxants. The diaphragm and laryngeal muscles are the most resistant to NMBAs. However, onset and offset times are relatively rapid as a result of high regional blood flows. The onset, intensity, and duration of neuromuscular block are relatively similar in the muscles of the diaphragm, the larynx, and the orbital muscles surrounding the eye. Profound relaxation of the laryngeal muscles is required to facilitate tracheal intubation. Good intubating conditions can be predicted with more confidence if the orbicularis oculi ${ }^{27}$ or corrugator superciliii ${ }^{28}$ muscles surrounding the eye are monitored, because these muscles reflect the extent of neuromuscular block of the laryngeal muscles. The peripheral muscles of the limbs and the upper airway musculature are the most sensitive to NMBAs and take the longest time to recover. Because the adductor pollicis is one of the last muscles to recover from neuromuscular blockade, it is essential to monitor this site to ensure full recovery of neuromuscular function.

\section{Intense Neuromuscular Blockade}

Intense muscle relaxation is needed to guarantee a smooth tracheal intubation. In rare circumstances, profound neuromuscular blockade is required to prevent patient movement (ie, during certain ophthalmic or neurosurgical procedures). Patient movement during intubation or with surgical stimulation can still occur when a TOF count of 0 at the adductor pollicis is observed. Two methods have been described to evaluate deep neuromuscular block. Fernando et al. examined the use of posttetanic count monitoring in predicting coughing or bucking in response to carinal stimulation. ${ }^{29}$ To completely eliminate any response to carinal stimulation, a PTC of 0 at the adductor pollicis was needed. Severe responses occurred when the PTC was greater than 2 or 3 . A second method used to evaluate intense neuromuscular block involves the monitoring of a muscle that is relatively resistant to NMBAs such as the orbicularis oculi. Bucking in response to tracheal intubation is unlikely to occur when a TOF count of 0 at the orbicularis oculi is measured, because the orbicularis oculi and diaphragm require larger concentrations of NMBAs to achieve the same blockade as the adductor pollicis.

\section{Moderate Neuromuscular Block}

During any surgical procedure, the smallest dose of a NMBA that will provide optimal surgical conditions should be used. An over reliance on 
muscle relaxants to prevent patient movement in the operating room can contribute to residual paralysis in the postoperative period. Adequate surgical relaxation during abdominal surgery is present when greater than $80 \%$ depression of a single twitch is achieved. ${ }^{30}$ This corresponds to 1 to 2 twitches in the TOF count. Maintaining a TOF count of 1 to 2 is appropriate during most surgical procedures in the presence of inhalational agents. Patient movement is still possible at this level of neuromuscular blockade, however.

\section{Reversal of Neuromuscular Block}

The ability to successfully antagonize a neuromuscular block is dependent on the intensity of the block at the time of reversal. If a TOF count of 4 is present at the end of surgery, most nondepolarizing muscle relaxants can be fully reversed within 10 minutes. ${ }^{31}$ When only one response to TOF stimulation is observed, adequate reversal (TOF ratio $>0.7$ ) could take as long as 20 to 30 minutes. ${ }^{32}$ Antagonism of a block should not be attempted if a TOF count of 0 is measured. Administering anticholinesterase agents at this time will not accelerate recovery. Spontaneous recovery to a TOF count of 1 to 2 should occur before reversal drugs are administered.

Monitoring recovery of neuromuscular function residual paralysis after the use of nondepolarizing muscle relaxants is a relatively common occurrence in the early postoperative period. One of the most important applications of neuromuscular monitoring in the perioperative setting is its use in assessing, and possibly preventing, residual weakness in the recovery room and intensive-care unit.

\section{Assessing Residual Weakness in the Perioperative Period}

Small degrees of neuromuscular block can be difficult to assess on clinical examination before and after extubation. A visual or tactile evaluation of TOF responses is an unreliable method of detecting residual paresis when the TOF ratios are greater than 0.4. Several studies have demonstrated that DBS is more sensitive than the TOF pattern of stimulation in detecting fade when the TOF ratio is between 0.4 and $0.6{ }^{19,33}$ However, when the TOF ratio is 0.7 , there is still a $47 \%$ chance that fade will not be recognized by experienced clinicians when $\mathrm{DBS}_{3,3}$ monitoring is used. ${ }^{19}$ The only type of monitoring that will accurately measure subtle degrees of neuromuscular block are mechanomyography, electromyography, or acceleromyography. At the present time, only acceleromyography units are commercially produced for clinical use. Mechanomyography and electromyography monitoring is usually limited to research purposes, because these devices are bulky, difficult to calibrate, and subject to interference by factors in the operating room. 


\section{Does a Train-of-Four Ratio of 0.7 Represent Adequate Recovery of Neuromuscular Function?}

Traditionally, a TOF ratio of at least 0.7 has been considered to reflect sufficient recovery of muscle strength to allow extubation of the trachea. A TOF ratio of 0.7 has been associated with the ability to maintain a 5 -second head lift in the majority of patients, ${ }^{34}$ a normal tidal volume, a vital capacity of 15 to $20 \mathrm{~mL} / \mathrm{kg},{ }^{35}$ and a maximal inspiratory pressure of greater than $-20 \mathrm{~cm} \mathrm{H}_{2} 0 .{ }^{1,35}$ Several recent studies have suggested that a TOF ratio of 0.7 does not represent adequate recovery of neuromuscular function, and that signs and symptoms of muscle weakness persist until the TOF ratio is greater than 0.8 or 0.9 . Pharyngeal function during partial neuromuscular blockade has been studied in volunteers. ${ }^{36}$ At TOF ratios of 0.7 and 0.8 , significant pharyngeal dysfunction, misdirected swallowing, and episodes of aspiration were observed. Normal pharyngeal function was restored only when TOF ratios of greater than 0.9 were recorded. Eriksson et al. demonstrated that TOF ratios of 0.7 were associated with significant impairment of the hypoxic ventilatory response. ${ }^{37}$ This was attributed to a direct effect of nondepolarizing muscle relaxants on the carotid body. A significantly higher incidence of hypoxemia has been reported in postoperative patients with residual paresis. ${ }^{38}$ Hypoxemia occurred in $60 \%$ of the patients who had a measured TOF ratio less than 0.7 on admission to the recovery room. Only $10 \%$ of the patients who had TOF ratios greater than 0.7 exhibited hypoxemia during the early recovery period. Berg et al. recently demonstrated that residual neuromuscular block is a risk factor for postoperative pulmonary complications. ${ }^{39}$ TOF ratios were measured on arrival to the recovery room in patients randomized to receive pancuronium, atracurium, or vecuronium intraoperatively. In the pancuronium group, significantly more patients with TOF ratios less than 0.7 developed postoperative atelectasis or pneumonia $(16.9 \%)$ compared with subjects without residual block $(4.8 \%)$. The subjective experience of small degrees of residual neuromuscular block could be highly unpleasant for the patient after surgery. The clinical signs and symptoms of residual paralysis in awake volunteers have been recently described. ${ }^{40}$ TOF ratios of 0.7 to 0.75 were associated with diplopia and visual disturbances, severe facial weakness, difficulty speaking, an inability to maintain incisor teeth apposition, an inability to sit up alone, decreased grip strength, and generalized fatigue. Taken together, all of these studies indicate that a TOF ratio of greater than or equal to 0.9 is required to ensure a full clinical recovery of neuromuscular function (Table 1).

\section{Intraoperative Neuromuscular Monitoring and Postoperative Residual Paresis}

The use of long-acting nondepolarizing NMBAs is clearly associated with a high incidence of postoperative residual paralysis. When pancuro- 
Table 1. Risks Associated With Residual Neuromuscular Blockade in the Postoperative Period

Pharyngeal dysfunction and aspiration

Impairment of the hypoxic ventilatory response

Postoperative hypoxemia

Increased incidence of atelectasis and pneumonia

Patient discomfort (visual disturbances, facial weakness, difficulty speaking and swallowing, inability to sit up)

Prolonged recovery times in the postanesthesia care unit

nium is used in the operating room, TOF ratios $<0.7$ are measured in $36 \%$ to $47 \%$ of patients in the immediate recovery period. ${ }^{39,41,42}$ The incidence of residual paresis (TOF $<0.7$ ) is reduced to $4 \%$ to $15 \%$ when intermediate-acting muscle relaxants are used intraoperatively. ${ }^{39,41,42}$ Several prospective, randomized clinical studies have examined the influence of intraoperative neuromuscular monitoring on the occurrence of residual muscle weakness after surgery. Pedersen et al. investigated the effect of intraoperative TOF monitoring, using a tactile evaluation of evoked responses, on the incidence of postoperative residual paresis. ${ }^{43}$ An equal number of patients in the monitored (12 of 20) and unmonitored (12 of 20) groups had TOF ratios $<0.7$ on arrival to the recovery room. In contrast to these findings, Shorten et al. reported that a manual evaluation of TOF responses in surgical patients significantly reduced the occurrence of residual muscle weakness. ${ }^{44}$ At the conclusion of surgery, TOF ratios $<0.7$ were found in $47 \%$ of the unmonitored patients and in only $15 \%$ of the monitored patients. The incidence and degree of residual block could also be decreased when DBS is used perioperatively. In comparison to a group of subjects in whom no neuromuscular monitoring was used, the routine application of $\mathrm{DBS}_{3,3}$ monitoring resulted in significant fewer patients $(57 \%$ vs. $24 \%)$ exhibiting postoperative residual paralysis. ${ }^{33}$ The serious problem of residual neuromuscular block can virtually be eliminated if acceleromyography is used in the operating room and tracheal extubation not attempted until TOF ratios are $>0.7 .^{45}$ In this study, improvements in clinical recovery occurred at the expense of a slightly longer time ( 5 minutes) from the end of the operation to extubation. In summary, it appears that the routine use of neuromuscular monitoring, in particular DBS and acceleromyography, can play an important role in attenuating the incidence and severity of residual paralysis in the early recovery period.

\section{- Conclusion}

The response of an individual patient to a neuromuscular-blocking agent can be highly variable. The use of a peripheral nerve stimulator in 
the perioperative period allows muscle relaxants to be titrated to each patient's unique clinical requirements. Neuromuscular monitoring should be used whenever NMBAs are administered to ensure optimal patient care.

\section{- References}

1. Ali H, Savarese J. Monitoring of neuromuscular function. Anesthesiology. 1976;45:216221.

2. Van der Kloot W, Molgo J. Quantal acetylcholine release at the vertebrate neuromuscular junction. Physiol Rev. 1994;74:899-902.

3. Gibb AJ, Marshall IG. Pre- and post-junctional effects of tubocurarine and other nicotinic antagonists during repetitive stimulation in the rat. J Physiol. 1984;351:275-280.

4. Thesleff $S$. The mode of neuromuscular block caused by acetylcholine, nicotine, decamethonium, and succinylcholine. Acta Physiol Can. 1955;34:218-222.

5. Beemer GH, Reeves JH, Bjorksten AR. Accurate monitoring of neuromuscular blockade using a peripheral nerve stimulator-a review. Anaesth Intensive Care. 1990;18:490496.

6. Kopman AF, Lawson D. Milliamperage requirements for supramaximal stimulation of the ulnar nerve with surface electrodes. Anesthesiology. 1984;61:83-85.

7. Cooper JB, DeCesare R, D'Ambre MN. An engineering critical incident: direct current burn from a neuromuscular stimulator. Anesthesiology. 1990;73:168-170.

8. Bevan DR, Donati F. Muscle relaxants. In: Barash PG, Cullen BF, Stoelting RK, eds. Clinical Anesthesia, ed 4. Philadelphia: Lippincott Williams and Wilkins; 2001:419-447.

9. Ali $\mathrm{HH}$, Saverese JJ. Stimulus frequency and dose-response curve to d-tubocurarine in man. Anesthesiology. 1980;52:36-39.

10. Lee C. Train-of-four quantitation of competitive neuromuscular block. Anesth Analg. 1975;54:649-653.

11. Ali HH, Utting JE, Gray TC. Quantitative assessment of residual antidepolarizing block (part 2). Br J Anaesth. 1971;43:478-485.

12. Merton PA. Voluntary strength and fatigue. J Physiol (Lond). 1954;123:553-564.

13. Kopman A, Epstein R, Flashberg M. Use of $100 \mathrm{~Hz}$ tetanus as an index of recovery from pancuronium-induced non-depolarizing neuromuscular blockade. Anesth Analg. 1982; 61:439-442.

14. Brull SJ, Silverman DG. Tetanus-induced changes in apparent recovery after bolus doses of atracurium or vecuronium. Anesthesiology. 1992;77:642-649.

15. Viby-Mogensen J, Howardy-Hansen P, Chraemmer-Jorgensen B, et al. Post-tetanic count (PTC). A new method of evaluating an intense nondepolarizing neuromuscular blockade. Anesthesiology. 1981;55:458-462.

16. Bonsu AK, Viby-Mogensen J, Fernando PUE, et al. Relationship of post-tetanic count and train-of-four response during intense neuromuscular blockade caused by atracurium. BrJ Anaesth. 1987;59:1089-1091.

17. Muchhal KK, Viby-Mogensen J, Fernando PUE, et al. Evaluation of intense neuromuscular blockade caused by vecuronium using post-tetanic count (PTC). Anesthesiology. 1987;66:846-850.

18. Brull SJ, Connelly NR, Silverman DG. Correlation of train-of-four and double-bursts stimulation ratios at varying amperages. Anesth Analg. 1990;71:489-491.

19. Drenck NE, Ueda N, Olsen NV, et al. Manual evaluation of residual curarization using double burst stimulation: a comparison with train-of-four. Anesthesiology. 1989;70:578581. 
20. Viby-Mogensen J, Jensen NH, Engbaek J, et al. Tactile and visual evaluation of the response to train-of-four stimulation. Anesthesiology. 1985;63:440-443.

21. Kalli I. Effect of surface electrode position on the compound action potential evoked by ulnar nerve stimulation during isoflurane anesthesia. Br J Anaesth. 1990;65:494-499.

22. Engbaek J, Ostergaard D, Viby-Mogensen J. Clinical recovery and train-of-four ratio measured mechanically and electromyographically following atracurium. Anesthesiology. 1989;71:391-395.

23. May O, Kirkegaard NH, Werner MU. The acceleration transducer-an assessment of its precision in comparison with a force displacement transducer. Acta Anaesthiol Scand. 1988;32:239-243.

24. Itagaki T, Tai K, Katsumata N, et al. Comparison between a new acceleration transducer and a conventional force transducer in the evaluation of twitch responses. Acta Anaesthiol Scand. 1988;32:347-349.

25. Dascalu A, Geller E, Moalem Y, et al. Acoustic monitoring of intraoperative neuromuscular block. Br J Anaesth. 1999;83:405-409.

26. Bellemare F, Couture J, Donati F, et al. Temporal relation between acoustic and force responses at the adductor pollicis during nondepolarizing neuromuscular block. Anesthesiology. 2000;93:646-652.

27. Donati F, Meistelman C, Plaud B. Vecuronium neuromuscular blockade at the diaphragm, the orbicularis oculi, and adductor pollicis muscles. Anesthesiology. 1990;73: 870-875.

28. Plaud B, Debaene B, Donati F. The corrugator supercilii, not the orbicularis oculi, reflects rocuronium neuromuscular blockade at the laryngeal adductor muscles. Anesthesiology. 2001;95:96-101.

29. Fernando PUE, Viby-Mogensen J, Bonsu AK, et al. Relationship between post-tetanic count and response to carinal stimulation during vecuronium-induced neuromuscular blockade. Acta Anaesthesiol Scand. 1987;31:593-597.

30. deJong RH. Controlled relaxation 1. Quantitation of EMG with abdominal relaxation. JAMA. 1966;197:393-397.

31. Viby-Mogensen J. Neuromuscular monitoring. In: Miller RD, ed. Anesthesia, ed 4. New York: Churchill Livingstone; 2000:1351-1366.

32. Kopman AF. Recovery times following edrophonium and neostigmine reversal of pancuronium, atracurium, and vecuronium steady-state infusions. Anesthesiology. 1986;65: $572-576$.

33. Fruergaard K, Viby-Mogensen J, Berg H, et al. Tactile evaluation of the response to double burst stimulation decreases, but does not eliminate, the problem of postoperative residual paralysis. Acta Anaesthiol Scand. 1998;42:1168-1174.

34. Brand JB, Cullen DJ, Wilson NE, et al. Spontaneous recovery from nondepolarizing neuromuscular blockade: Correlation between clinical and evoked responses. Anesth Analg. 1977;56:55-58.

35. Dupuis JY, Martin R, Tetrault JP. Clinical, electrical and mechanical correlations during recovery from neuromuscular blockade with vecuronium. Can J Anaesth. 1990;37:192198.

36. Sundman E, Witt H, Olsson R, et al. The incidence and mechanisms of pharyngeal and upper esophageal dysfunction in partially paralyzed humans. Anesthesiology. 2000;92: 977-984.

37. Eriksson LI, Sato M, Severinghaus JW. Effect of a vecuronium-induced partial neuromuscular block on hypoxic ventilatory response. Anesthesiology. 1993;78:693-699.

38. Bissinger U, Schimek F, Lenz G. Postoperative residual paralysis and respiratory status: a comparative study of pancuronium and vecuronium. Physiol Res. 2000;49:455-462.

39. Berg H, Viby-Mogensen J, Roed J, et al. Residual neuromuscular block is a risk factor for postoperative pulmonary complications. A prospective, randomized, and blinded 
study of postoperative pulmonary complications after atracurium, vecuronium and pancuronium. Acta Anaesthesiol Scand. 1997;41:1095-1103.

40. Kopman AF, Yee PS, Neuman GG. Relationship of the train-of-four fade ratio to clinical signs and symptoms of residual paralysis in awake volunteers. Anesthesiology. 1997;86: $765-771$.

41. Bevan DR, Smith CE, Donati F. Postoperative neuromuscular blockade. A comparison between atracurium, vecuronium and pancuronium. Anesthesiology. 1988;69:272-276.

42. Anderson BN, Madsen JV, Schurizek BA, et al. Residual curarisation: a comparative study of atracurium and pancuronium. Acta Anaesthesiol Scand. 1988;32:79-81.

43. Pedersen T, Viby-Mogensen J, Bang U, et al. Does perioperative tactile evaluation of the train-of-four response influence the frequency of postoperative residual neuromuscular blockade? Anesthesiology. 1990;73:835-839.

44. Shoten GD, Merk H. Perioperative train-of-four monitoring and residual curarization. Can J Anaesth. 1995;42:711-715.

45. Mortensen CR, Berg H, El-Mahdy A. Perioperative monitoring of neuromuscular transmission using acceleromyography prevents residual neuromuscular block following pancuronium. Acta Anaesthesiol Scand. 1995;39:797-801. 